\title{
Berry's Paradox... Again
}

\author{
Graham Priest
}

Departments of Philosophy, the CUNY Graduate Center and the University of Melbourne

\begin{abstract}
The paper is a discussion of whether Berry's Paradox presupposes the Principle of Excluded Middle, with particular reference to the work of Ross Brady.
\end{abstract}

\section{Introduction}

On and off over the years, Ross Brady and I have engaged in a dialogue on the question of Berry's Paradox and the Principle of Excluded Middle $(P E M){ }^{1}$ What is at issue is whether the paradox can be avoided if one gives up the $P E M$. I say that it can not; he says that it can. ${ }^{2}$ This is important for the following reason. I endorse a solution to the paradoxes of self-reference rejecting Explosion. He endorses a solution rejecting the PEM. If I am right, then his solution cannot handle Berry's paradox, whilst mine can. Brady's most recent contribution to the debate is his (2017). This paper is mine. ${ }^{3}$

\section{Berry's Paradox}

Berry's Paradox is familiar enough to need little introduction. English, with its current vocabulary, has an infinite number of (non-indexical) referential

\footnotetext{
${ }^{1}$ E.g., Priest (1983), Brady (1984), Priest (1987), ch. 1.

${ }^{2}$ See Brady (2017), $\S 7$.

${ }^{3}$ The considerations in what follows generally extend to König's paradox. However, I will not go into these matters here,
}

Australasian Journal of Logic (16:2) 2019, Article no. 2 
singular terms (names or descriptions). However, the vocabulary is finite, so the number of terms with less than, say 1,000 words is finite. Since the number of natural numbers is infinite there must be numbers that are not referred to by any of these, and so a least. By definition, this cannot be referred to by a term with less than 1,000 words. But 'the least natural number not referred to by a term with less that 1,000 words' has less than 1,000 words, and this refers to it.

A formal reconstruction of the argument can be carried out in first-order arithmetic (with a finite number of variables, to ensure that the number of terms of any finite length is finite), augmented by a binary denotation predicate, $D$, and a description operator, $\mu$. One may think of the operator as either an indefinite description operator (an $x$ such that), or a least number operator (the least $x$ such that); it makes not difference. $D$ and $\mu$ satisfy the following conditions:

- $[D$ Schema $]\langle t\rangle x \leftrightarrow x=t$

- [Description Schema] $\exists x A(x) \rightarrow A(\mu x A)$

In the first of these, $t$ is any (closed) term, and $\langle t\rangle$ is the numeral of its gödel code, which functions as the name of $t$. In the second, bound variables in $A$ are are relabelled to avoid any clash when $\mu x A$ is substituted for $x$. We do not assume that a $\mu$-term denotes. How it behaves when no $x$ satisfies $A(x)$ is of no importance to the argument

The paradox argument now proceeds in two stages. The first applies the following principle of inference (where $I$ is some finite set):

$$
\begin{aligned}
& A(x) \leftrightarrow \bigvee_{i \in I} x=n_{i} \\
& \frac{(A(x) \wedge B(x, y) \wedge B(x, z)) \rightarrow y=z}{\exists y \neg \exists x(A(x) \wedge B(x, y))}
\end{aligned}
$$

The first premise says that the extension of $A(x)$ comprises the $n_{i} s$, and so is finite. The second says that for any $x$ satisfying $A(x)$ there is at most one $y$ such that $B(x, y)$. The conclusion says that there is some $y$ which is not related by $B(x, y)$ to any $x$ satisfying $A(x)$.

This inference records a purely combinatorial fact about numbers. That is, if $X$ is a finite subset of the natural numbers, and $f$ is a partial function with domain $X$, then the range of $f$ cannot contain all numbers. Its validity 
has nothing to do with any particular $X$ and $f$. The correctness of such purely arithmetic reasoning is not a contested matter in this debate.

Now, take for $A(x)$ the formula $M(x)$ which says that $x$ is a term of the language with at most 1,000 symbols. This is a purely arithmetic predicate, and the number of things which satisfy it is finite, say $n_{1}, \ldots, n_{k}$. Hence, the first premise is true. For $B(x, y)$ take $D x y$. If $M(x)$ then $x$ is some term, $t$. That is, $M(\langle t\rangle)$. And the $D$ Schema tells us that if $D\langle t\rangle y$ and $D\langle t\rangle z$ then $y=t=z$. Hence we have the second premise.

Given the inference, we have the conclusion:

- $\exists y \neg \exists x(M(x) \wedge D x y)$

That is, something is not denoted by any $x$ satisfying $M(x)$. This is the end of the first stage of the argument.

For the second stage, let $\tau$ be $\mu y \neg \exists x(M(x) \wedge D x y)$. Then by the result of the first stage, and the Description Schema:

- $\neg \exists x(M(x) \wedge D x \tau)$

However, provided that $M(x)$ is expressed in a suitably succinct way, $M(\langle\tau\rangle)$ is a simple arithmetic truth. Moreover, $\tau=\tau$, so by the $D$ Schema, $D\langle\tau\rangle \tau$. (No problems are posed by the possibility that ' $\tau$ ' may not have a denotation, since the result of the first stage of the argument guarantees that it has one.). Hence, $M(\langle\tau\rangle) \wedge D\langle\tau\rangle \tau$, and so $\exists x(M(x) \wedge D x \tau)$, contradicting what was established in the first stage.

There is no evident appeal to the PEM in all this.

\section{Identity}

One might suspect that the PEM has been somehow smuggled in to the above argument. To show that this is not so is precisely the point of a full formalisation I gave. The argument is formalised in a weak relevant logic without the $P E M$, say the logic $B .^{4}$ I note that the formalisation is given for $M(x)$ and $D x y$, but can be used to establish the conclusion of the first section of the argument of the previous section for any $A(x)$ and $B(x, y)$ satisfying the premises of the general inference deployed there.

\footnotetext{
${ }^{4}$ See, e.g., Priest (2008), $\S \S 10.1-10.3$.
} 
This argument by has been criticised by Brady. ${ }^{5}$ His objection is to the identity principle employed in the proof:

- $[S](A(a) \wedge a=b) \rightarrow A(b)$

Now, the rejection of this principle, which is endorsed by most logicians, requires a good independent argument. Otherwise the rejection is ad hoc, and no better than simply rejecting any other step in the argument.

What, then, are Brady's reasons? He has two. The first is that $[S]$ is similar to the Pseudo-Modus-Ponens Principle $(P M P),(A \wedge(A \rightarrow B)) \rightarrow B$, which he and I both reject for reasons connected with Curry's Paradox. ${ }^{6}$ This is simply an argument of guilt by association, and carries no real weight. The second is that to derive $[S]$ from the substitutivity principle which Brady does accept (namely, $a=b, A(a) \vdash A(b)$ ) one needs principles such as $P M P$, or other principles which both he and I reject. Again, the fact that there are unacceptable proofs of something does not show that it is not true.

If one assumes that relevance is a virtue in a logic - or at least, if one assumes that irrelevance is a vice - then one might suggest that the addition of $[S]$ delivers a violation of relevance. And it is certainly true that some identity principles can deliver such violations. Thus, consider the principle $a=b \rightarrow(A(a) \rightarrow A(b))$. Choose $A$ to be any sentence with no free variables, and no occurrences of $a$ or $b$. Then $a=b \rightarrow(A \rightarrow A)$, and this certainly seems to be a violation of relevance, since the antecedent appears to have no bearing on the consequent. However, this is clearly not the case with $[S]$ : $(A \wedge a=b) \rightarrow A$ is not an intuitive violation of relevance.

Moreover, it can be shown that first-order constant-domain $B$, with identity governed by $[S]$ is relevant, in the sense that if $A \rightarrow B$ is a logical truth, $A$ and $B$ must have some predicate in common. ${ }^{7}$

In any case, one does not need the full strength of $[S]$ to carry out the

\footnotetext{
${ }^{5}$ Brady (2017), §7.

${ }^{6}$ See Priest (1987), ch. 6.

${ }^{7}$ The proof extends that given for propositions logic in Priest (2008), p. 220, ex. 11. For part (a), we simply take the extension of every $n$-place predicate at $\perp$ to be the set of all $n$-tuples from the domain, and the anti-extension of every $n$-place predicate at $\perp^{*}$ to be the empty set. This can be achieved for $=$ by taking the domain to be $\{a\}$, for some $a$. This also satisfies the constraint required for $[S]$. The rest of the proof is straightforward. I presume that the proof can be extended to stronger relevant logics by ensuring that the ternary $R$ satisfies the appropriate constraints.
}

Australasian Journal of Logic (16:2) 2019 Article no. 2 
proof. ${ }^{8}$ As an inspection of the proof shows, however, only one application of $[S]$ is made. This is of the form:

- $(y=\langle t\rangle \wedge D y x) \rightarrow D\langle t\rangle x$

This follows from:

- $(y=z \wedge z=x) \rightarrow y=z$

by the $D$ Schema and the Substitutivity of Equivalents, which is valid in the logic $B$. And Brady himself endorses this version of the transitivity of identity for numbers! ${ }^{9}$

Finally, let us note the following. None of this has anything to do with the $P E M$ ! Brady's reason for the Berry Paradox failing is that $[S]$ fails; and $[S]$ is a quite different principle. ${ }^{10}$ Since Berry's paradox is fairly uncontentiously classified as in the same family as paradoxes such as the Liar, Brady's solution fails because of the Principle of Uniform Solution: Same kind of paradox, same kind of solution. ${ }^{11}$

\section{The Description Schema}

An objection to the formalisation of Berry's paradox, on quite different grounds, has been given by Hartry Field, who also espouses a solution to the paradoxes of self-reference rejecting the PEM. Field targets the De-

\footnotetext{
${ }^{8}$ And indeed, one might well have reservations about any version of substitutivity within the scope of a conditional. See, for example, Priest (2008), §19.5.4-19.5.7.

${ }^{9}$ See Brady (2006), p. 314, and (2012), p. 361. I note that, oddly, nothing much is said about identity in general in Brady's (2006), though $(y=z \wedge z=x) \rightarrow y=z$ is a homomorph of $((A \rightarrow B) \wedge(B \rightarrow C)) \rightarrow(A \rightarrow C)$, which is an axiom of Brady's preferred logic (p. 6). The identity principle also holds for sets, given this propositional principle and the definition of identity (p. 174).

${ }^{10}$ The situation seems to have arisen for the following reason. In Priest (1983) I gave a formalisation of Berry's Paradox which assumed that every descriptive term denotes. Brady (1984) argued, plausibly, that this presupposes the $P E M$. The formalisation in Priest (1987) does not make this assumption. Hence, Brady's critique had to change tack.

${ }^{11}$ See Priest (1995), §11.5. The matter is different from that concerning Curry's Paradox, which both Brady and I wish to solve by rejecting $P M P$ and its like. Whether Curry's Paradox is in the same family as paradoxes such as the Liar is a contentious matter. See Priest (2017), $§ 15$.
}

Australasian Journal of Logic (16:2) 2019 Article no. 2 
scription Schema, where $\mu$ is the least number operator, on the ground that this implies the PEM, and so should be rejected if one rejects the $P E M{ }^{12}$

Field's argument goes as follows. Let $B$ be an arbitrary sentence, and let $A(x)$ be $x=1 \vee(x=0 \wedge B)$. Now clearly, $A(1)$, and so $\exists x A(x)$. Let $\tau$ be $\mu x A(x)$. The the Description Schema gives $A(\tau)$; that is, $\tau=1 \vee(\tau=0 \wedge B)$. This entails that $\tau=1 \vee \tau=0$. If $\tau=0$ then we can rule out the first disjunct, and so $B$ follows. If $\tau=1$, then $\neg A(0)$, since this is the least $n$ such that $A(n)$. That is, $\neg(0=1 \vee(0=0 \wedge B))$, which, given that $0=0$, entails $\neg B$.

Now, if there are truth-value gaps, this argument fails. For that 1 is the least $n$ such that $A(n)$ does not imply $\neg A(0)$, since $A(0)$ may simply be neither true nor false. Indeed, assuming that the extensional connectives work in the standard way (say of $K_{3}$ ), if $B$ is neither true nor false, so is $A(0)$. Hence, the argument presupposes that $B \vee \neg B$; so Field's argument begs the question. For good measure, as already noted, the formalistion of Berry's paradox can be run where the description operator is an indefinite description operator, $\varepsilon$. Then the fact that $\tau=1$ has no implications for $A(0)$ at all.

Indeed, given an interpretation of the language of arithmetic which allows for the possibility that some sentences are neither truth nor false, this can be extended to an interpretation for the least number operator. $\mu x A(x)$ denotes the least number, $n$, such that $A(n)$, if there is such; and otherwise either denotes 0 or is undefined (depending on how one wants to treat the other case). This verifies the Description Schema, and is a conservative extension, which does not, therefore, deliver the PEM. Similarly, for nearly all standard logics (including $B$ ), given an interpretation of the language, this can be extended to an interpretation for an indefinite description operator. $\varepsilon x A(x)$ denotes an object in the domain, $n$, picked out by a choice function, such that $A(n)$, if there is such; and otherwise either denotes some fixed but arbitrary object or is undefined (depending on how one wants to treat the other case). This verifies the Description Schema, and is a conservative extension. So if the $P E M$ is not valid in the original interpretation, the Description Schema does not deliver it. ${ }^{13}$

In his (2008), Field also argues against the Description Schema for a leastnumber operator, on the ground that it would imply the existence of a cut-off

\footnotetext{
${ }^{12}$ See Field (2005), esp. fn 14, and (2008). The points below come from Priest (2005) and (2010).

${ }^{13}$ That the Description Schema for indefinite descriptions does not imply PEM in the context of intutitionist logic is shown in Bell (1993).
}

Australasian Journal of Logic (16:2) 2019 Article no. 2 
point in sorites sequences, which is counter-intuitive. This is not relevant to the present case, since the counter-example concerns vague predicates, and numerical predicates are not vague. And in any case, the point does not apply to an indefinite description operator.

\section{Conclusion}

We have now looked at a number of arguments as to why Berry's paradox presupposes the PEM. I have argued that these do not succeed. Berry's paradox, then, still shows that the paradoxes of self-reference cannot be avoided by giving this up. ${ }^{14}$

\section{References}

[1] Beall, J. C., and Armour-Garb, B. (eds.) (2005), Deflationism and Paradox, Oxford: Oxford University Press.

[2] Bell, J. (1993), 'Hilbert's ع-Operator and Classical Logic, Journal of Philosophical Logic 22: 1-18.

[3] Brady, R. (1984), 'Reply to Priest on Berry's Paradox', Philosophical Quarterly 34: 157-63.

[4] Brady, R. (2006), Universal Logic, Stanford, CA: CSLI Publications.

[5] Brady, R. (2012), 'The Consistency of Arithmetic, Based on a Logic of Meaning Containment', Logique et Analyse 219: 353-83.

[6] Brady, R. (2017), 'The Use of Definitions and their Logical Representation in Paradox Derivation', Synthese, DOI: 10.1007/s11229-017-1362-7.

[7] Field, H. (2005), 'Is the Liar Both True and False?', ch. 2 of Beall and Armour-Garb (2004).

[8] Field, H. (2008), Saving Truth From Paradox, Oxford: Oxford University Press.

\footnotetext{
${ }^{14}$ This paper was given at a workshop at the Univerisy of Melbourne in honour of Brady's work, in August 2018. Many thanks go to the audience there for their helpful comments, and especially to Ross Brady himself, Lloyd Humberstone, Ed Mares, and Greg Restall.
}

Australasian Journal of Logic (16:2) 2019 Article no. 2 
[9] Priest, G. (1983), 'Logical Paradoxes and the Law of Excluded Middle', Philosophical Quarterly 33: 160-5.

[10] Priest, G. (1987), In Contradiction, Dordrecht: Martinus Nijhoff; 2nd edn, Oxford: Oxford University Press, 2006.

[11] Priest, G. (1995), Beyond the Limits of Thought, Cambridge: Cambridge University Press; 2nd edn, Oxford, Oxford University Press, 2002.

[12] Priest, G. (2005), 'Spiking the Field Artillery', ch. 3 of Beall and Armour-Garb (2005).

[13] Priest, G. (2008), Introduction to Non-Classical Logic, 2nd edn, Cambridge: Cambridge University Press.

[14] Priest, G. (2010), 'Hopes Fade for Saving Truth', Philosophy 85: 109140.

[15] Priest, G. (2017), 'What If: the Exploration of an Idea', Australasian Journal of Logic 14, https://ojs.victoria.ac.nz/ajl/article/view/4028/3574.

Australasian Journal of Logic (16:2) 2019 Article no. 2 